\title{
Role of sterile pyuria in association to elevated PSA values in the diagnosis of non-palpable prostate cancer?
}

\author{
Selamettin Demir \\ Department of Urology, The Ministry of Health, University of Health Sciences, Van Education and Research Hospital, Van, Turkey.
}

\begin{abstract}
Summary Objectives: Although cancer is believed to develop and progress with the involvement of inflammation, it is still unclear what the correlation between inflammation and prostate cancer is. This study based on results of transrectal ultrasound-guided prostate biopsies aimed to determine whether $C$-reactive protein (CRP) and sterile pyuria were clinically useful in the evaluation of patients with suspect of prostate cancer.

Materials and methods: This study is a cross-sectional prospective study of patients without clinical prostatitis symptoms. Characteristics of the 200 consecutive patients recruited were 3-20 $\mathrm{ng} / \mathrm{mL}$ value of serum prostate-specific antigen (PSA), normal digital rectal examination finding, and sterile urine culture result. All patients underwent 12-core prostatic biopsy. 163 of the 200 patients had benign prostatic hyperplasia confirmed through histology, while the residual 37 patients had prostate cancer. Patients with pre-treatment urinary leukocyte count $\leq 3 /$ high power field were categorized as non-pyuria, whilst those with pre-treatment urinary leukocyte count > 3/high power field were categorized as pyuria. The serum CRP level was also used to differentiate patients before the biopsy. Subgroups were compared regarding a number of clinical variables.

Results: Histology revealed that $70 \%$ of pyuria patients and $38.5 \%$ of non-pyuria patients presented inflammation $(p=0.001)$. The pyuria group exhibited significantly higher total PSA compared to the non-pyuria group $(p=0.044)$. The two groups did not differ significantly regarding cancer detection rate $(p=0.752)$. CRP groups were similar regarding cancer detection and histologically-detected inflammation rates. Conclusion: In patients with no evidence of clinical prostatitis, sterile pyuria should be considered as a cause of increased PSA. Although sterile pyuria cannot predict non-palpable prostate cancer, it should be taken into account in urological evaluation in order to demonstrate minute prostatic inflammation due to its simplicity, convenience and non-invasiveness.
\end{abstract}

KEY WORDS: Prostate cancer; Inflammation; Biopsy; C-reactive protein; Pyuria.

Submitted 16 February 2019; Accepted 2 April 2019

\section{INTRODUCTION}

In the US, prostate cancer (PCa) continues to be the cancer with the second highest mortality rate in men, despite the fact that the number of men dying because of it is not as high as the number of those dying with it for other causes $(1,2)$. PCa risk is believed to be heightened by genetic and environmental factors, but the exact etiology remains unclear (3). Nevertheless, PCa and partic- ularly non-palpable PCa has been increasingly identified at an early stage thanks to the common practice of screening for serum prostate-specific antigen (PSA). However, some have argued that biopsies should not be performed unnecessarily $(4,5)$, because, besides PCa, non-malignant prostate conditions, such as inflammation, may be associated with high serum PSA as well $(6,7)$. The general assumption is that PCa develops and progresses due to inflammatory processes that disrupt the function of oncogenes and tumor inhibitors, thus damaging DNA and activating processes that can trigger tumor cells to grow and proliferate $(8,9)$. Therefore, PCa risk is considered to be elevated in men with intraprostatic conditions like proliferative inflammatory atrophy, prostatic intraepithelial neoplasia, and prostatitis, which may be induced by acute or chronic inflammation and are believed to be PCa precursors $(9,10)$.

Abnormal digital rectal examination (DRE) results and/or PSA higher than $4.0 \mathrm{ng} / \mathrm{mL}$ are among the main markers warranting a prostate biopsy. If DRE suggests that PCa is likely, then biopsy is vital. On the other hand, PSA is the sole basis for deciding performance on non-palpable case biopsy, although it frequently yields false positives because its levels are increased by inflammation (11). Thus, it is important to prevent performing unnecessary biopsy for non-palpable case, and for this purpose, a number of PSA derived parameters have been considered, including free-to-total PSA ratio, PSA density (PSAD) and PSA velocity $(12,13)$.

This study sought to determine the extent to which the impact of inflammation on detection of cancer in specimens of prostate biopsy could be gauged based on serum C-reactive protein (CRP) level and urinary leukocyte count.

\section{MATERIALS AND METHOdS}

Ethics committee approval was received for this study from the hospita ethics committee (number: 218/13). Written informed consent was obtained from patients who participated in this study. A number of 200 consecutive patients were recruited for this study. All of them had serum PSA levels in the range 3-20 ng/mL, normal DRE findings and sterile urine culture results and underwent transrectal ultrasound-guided 12-core prostatic biopsy (TRUS-bx) beetwen Januray 2018 and February 2019. The study did not include patients with clinical signs of 
prostatitis, macroscopic pyuria, bacteria isolated in urine culture or systemic inflammatory conditions with potential impact on serum CRP. The medication regimen consisted of $500 \mathrm{mg}$ ciprofloxacin administered orally twice daily over a period of one week, with the first dose being given 24 hours before the procedure. Patients with pretreatment urinary leukocyte count $\leq 3 /$ high power field (h.p.f.) were categorized as non-pyuria, whilst those with pre-treatment urinary leukocyte count $>3 /$ h.p.f. were categorized as pyuria. Furthermore, a positive group with serum CRP of $0.30 \mathrm{mg} / \mathrm{dL}$ or higher and a negative group with serum CRP of less than $0.30 \mathrm{mg} / \mathrm{dL}$ were distinguished as well (25). Elecsys PSA kit (Roche Cobas 411, Tokyo, Japan) was used for measurement of free and total PSA values. Alongside the blood sample, urine culture and urine samples were also acquired prior to DRE. Latex CRP immunoturbidimetric kit (CRP-Latex 'CRPLX', Roche, Germany, and Model no. 6000; Hitachi, Tokyo, Japan) permitted measurement of serum CRP before treatment, with $0.30 \mathrm{mg} / \mathrm{dL}$ cut-off value with $93 \%$ specificity (25). Furthermore, a centrifugal automatic analyzer and a light microscope (Roche Miditron ${ }^{\circledR}$ Junior II, Germany) was used for urine analysis, while a scanner with a $6.5 \mathrm{MHz}$ transrectal probe enabled performance of prostate transrectal ultrasonography (TRUS). Prostate scanning was performed in both transverse and sagittal planes whilst the patient was lying on his left side. The formula for a prostate ellipsoid (width $\times$ length $\times$ height $\times$ $0.523)$ helped to calculate the prostate volume and the total PSA was divided by the prostate volume to obtain the PSAD. Moreover, an automatic biopsy gun and an 18-gauge needle were employed to conduct the 12-quadrant biopsy under TRUS guidance. Prostate chronic inflammation was considered to be present when inflammatory cells, lymphocytes, plasma cells and/or histiocytes infiltrated prostatic biopsy specimens (14)

Data were expressed as means \pm SD SPSS (IBM SPSS for Windows, ver.24) was used for comparison of the variables associated with the different groups via Student's t-test and ChiSquare test. Statistical significance was indicated by $\mathrm{P}$ value of less than 0.05 .

\section{RESULTS}

The characteristics of every recruited patient are shown in Table 1. A proportion of $81.5 \%$ of patients ( $n=163$ ) was histologically confirmed to have benign prostatic hyperplasia (BPH), while the other $18.5 \%$ of patients ( $\mathrm{n}=$ 37) had PCa. Histology also showed inflammation in biopsy specimens of a proportion of $49.5 \%$ of patients ( $\mathrm{n}=$ 99). A comparison of PCa and $\mathrm{BPH}$ patients in terms of clinical variables is provided in Table 1. PCa patients had a mean age of $65.47 \pm 7.84$ years, while BPH patients had a mean age of $63.12 \pm 7.73$ years $(p=0.197)$. PCa patients had significantly higher total PSA compared to BPH patients ( $\mathrm{p}$ $=0.001$ ), but the two groups were similar in terms of free-to-total PSA ratios. Furthermore, PCa patients had significantly lower mean prostate volumes $(p=0.040)$, the PSAD values were significantly higher in the PCa than in the BPH group $(p=0.001)$. However, histology did not show a significant difference between the groups regarding inflammation ( $\mathrm{p}=0.738)$.

A proportion of $35 \%$ of patients $(n=70)$ exhibited pyuria, while the other $65 \%(n=130)$ did not, as revealed by the distribution of the urinary leukocyte count. A comparison of these two groups in terms of clinical variables, cancer detection rates, and histologically-detected inflammation is provided in Table 2. The groups were similar regarding age, free-to-total PSA ratio, prostate volume, and PSAD value, although the pyuria group exhibited significantly higher total PSA compared to the non-pyuria group ( $\mathrm{p}=$ 0.044 ), as well as significantly higher serum CRP ( $p=$ 0.001 ). Furthermore, the groups were similar in terms of histological cancer detection rate $(p=0.752)$, but the pyuria group displayed greater histological inflammation (70\% vs $38.5 \%$; $\mathrm{p}=0.001$ ).
Table 1.

Characteristics of recruited patients.

\begin{tabular}{|c|c|c|c|}
\hline & $\mathrm{BPH}(\mathrm{n}=163)$ & $\mathrm{PCa}(n=37)$ & $\mathbf{P}$ \\
\hline Age (years) & $63.12 \pm 7.73(40-79)$ & $65.47 \pm 7.84(45-80)$ & 0.637 \\
\hline Urinary leukocyte count (nºh.p.f.) & $9.44 \pm 19.18(1-80)$ & $10.71 \pm 21.25(1-90)$ & 0.288 \\
\hline $\mathrm{CRP}(\mathrm{mg} / \mathrm{dL})$ & $1.23 \pm 1.55(0.10-8.00)$ & $0.85 \pm 0.63(0.10-3.12)$ & 0.520 \\
\hline T-PSA (ng/mL) & $5.84 \pm 2.54(3.11-14)$ & $8.72 \pm 4.48(3.65-20.00)$ & $0.001 *$ \\
\hline Prostate volume $(\mathrm{mL})$ & $57.77 \pm 34.65(17-260)$ & $41.2 \pm 20.36(20-120)$ & $0.040 *$ \\
\hline Free to total PSA ratio (\%) & $19 \pm 7(5-38)$ & $22 \pm 23(3-58)$ & 0.752 \\
\hline$\overline{\mathrm{PSAD}}$ & $0.12 \pm 0.07(0.03-0.34)$ & $0.25 \pm 0.21(0.07-0.85)$ & $0.001 *$ \\
\hline $\begin{array}{l}\text { Histologically-detected } \\
\text { inflammation rate }\end{array}$ & $49.7 \%(81 / 163)$ & $48.6 \%(18 / 37)$ & 0.738 \\
\hline
\end{tabular}

Table 2.

Clinical variables, cancer detection rates and histologically-detected inflammation rates in pyuria and non-pyuria groups.

\begin{tabular}{|c|c|c|c|}
\hline & Non-pyuria group (n: 130) & Pyuria group (n: 70) & p \\
\hline Age (years) & $60.45 \pm 7.51(40-80)$ & $64 \pm 6.07(50-78)$ & 0.637 \\
\hline $\mathrm{CRP}(\mathrm{mg} / \mathrm{dL})$ & $0.75 \pm 0.78(0.10-3.22)$ & $2.39 \pm 2.09(0.28-8.00)$ & $0.001 *$ \\
\hline T.PSA (ng/mL) & $6.42 \pm 2.41(3.29-15.07)$ & $7.85 \pm 3.88(3.11-20.00)$ & $0.044 *$ \\
\hline Prostate volume(mL) & $50.3 \pm 34.4(17-260)$ & $55.4 \pm 26.5(30-130)$ & 0.208 \\
\hline Free to total PSA ratio (\%) & $17 \pm 9(3-35)$ & $20 \pm 11(11-58)$ & 0.814 \\
\hline$\overline{P S A D}$ & $0.14 \pm 0.07(0.04-0.61)$ & $0.15 \pm 0.12(0.03-0.85)$ & 0.288 \\
\hline $\begin{array}{l}\text { Histologically-detected } \\
\text { inflammation rate }\end{array}$ & $38.5 \%(50 / 130)$ & $70 \%(49 / 70)$ & $0.001 *$ \\
\hline Cancer detection rate & $17.6 \%(23 / 130)$ & $20 \%(14 / 70)$ & 0.752 \\
\hline
\end{tabular}


Table 3.

Clinical variables, cancer detection rates and histologically-detected inflammation rates in positive- and negative- $C$-reactive protein groups.

\begin{tabular}{|lccc|}
\hline & CRP negative (n: 57) & CRP positive (n: 143) & P \\
\hline Age (years) & $63.15 \pm 7.20(40-77)$ & $60 \pm 6.37(43-80)$ & 0.245 \\
\hline T-PSA (ng/mL) & $6.80 \pm 2.45(3.23-14.58)$ & $7.15 \pm 3.63(3.11-20.00)$ & 0.991 \\
\hline Prostate volume(mL) & $55.10 \pm 37.32(17-120)$ & $59.88 \pm 29.53(20-260)$ & 0.200 \\
\hline Free to total PSA ratio (\%) & $19 \pm 7(3-55)$ & $16 \pm 8(4-58)$ & 0.738 \\
\hline PSAD & $0.15 \pm 0.06(0.03-0.35)$ & $0.17 \pm 0.12(0.04-0.85)$ & 0.490 \\
\hline Histologically-detected inflammation rate & $49.1 \%(28 / 57)$ & $49.6 \%(71 / 143)$ & 0.857 \\
\hline Cancer detection rate & $12.3 \%(7 / 57)$ & $20.1 \%(30 / 143)$ & 0.248 \\
\hline $\begin{array}{l}\text { Mean } \pm \text { SD range; } \text {;Negative, serum CRP < } 0.30 \mathrm{mg} / \mathrm{dL} ; \text { positive, serum CRP } \geq 0.30 \mathrm{mg} / \mathrm{dL} ; \text { CRP, C-reactive protein; } \\
\text { T-PSA, total prostate specific antigen; PSAD, PSA density. }\end{array}$ & & \\
\hline
\end{tabular}

Two groups of positive CRP $(71.5 \%, \mathrm{n}=143)$ and negative CRP $(28.5 \%, \mathrm{n}=57)$ were also distinguished based on the manner in which pre-treatment serum CRP levels were distributed. A comparison of these two groups in terms of clinical parameters, cancer detection rates, and histologically-detected inflammation is provided in Table 3. It was observed that the groups were similar regarding age, free-to-total PSA ratio, prostate volume, cancer detection rates, and histologically-detected inflammation and PSAD values.

\section{Discussion}

Since the first isolation of serine protease PSA from prostate epithelial cells that was accomplished by Wang et al. in 1979, PCa began to be diagnosed and treated based primarily on PSA measurement (15). There are significant challenges involved in differentiating PCa from $\mathrm{BPH}$, especially in cases with intermediate levels of PSA. Furthermore, PSA is not associated exclusively with PCa but is expressed by malignant as well as normal prostate glands.

Efforts to make prostate biopsy more efficient directed attention to a number of factors associated with PSA, including the free-to-total PSA ratio, PSAD and PSA velocity $(12,13)$. Particular focus was put on serum markers like p53 antibody and insulin-like growth factor 1 (IGF-1) with the purpose of enhancing positive predictive value $(16,17)$, which has also benefitted from PCa visualisation innovations, including magnetic resonance spectroscopic imaging, dynamic contrastenhanced magnetic resonance imaging, positron emission tomography and transrectal power Doppler imaging $(18,19)$. Reduction of negative biopsy rate as much as possible should be a key priority of management since prostate biopsy can entail a great deal of pain and could result in considerable morbidity.

Besides PCa, benign conditions like benign prostatic hyperplasia, prostatic manipulation, and even asymptomatic and chronic prostatitis are associated with elevated levels of serum PSA $(6,7)$. It is well-known that the development and progression of numerous cancers are associated with the risk factor of inflammation (20), with pre-malignant modifications and adenocarcinoma of the prostate having been found to be underpinned by inflammatory processes (21). Cytokines stimulating rate of prostatic inflammation (11).

inflammation, especially interleukin 6 (IL-6) (22), have a significant influence on systemic inflammation markers like CRP (23). However, IL-6 is not only involved in the mobilization of inflammatory cells but is also believed to be mitogenic in the case of prostate cells (24). In a retrospective study on 284 patients showing no cancer signs in sextant ultrasound-guided biopsies, Morote et al. reported that $23.2 \%$ of patients had non-malignant tissue without inflammation, $68.3 \%$ displayed chronic prostatitis, and $8.4 \%$ displayed acute prostatitis, which implied that BPH specimens had a high In a different retrospective study, Tomonori et al. found that $53.5 \%$ of $\mathrm{BPH}$ patients and $14.1 \%$ of $\mathrm{PCa}$ patients had inflammation (25).

In the present study, histology revealed that $49.7 \%$ of BPH patients and $48.6 \%$ of PCa patients had inflammation. Asymptomatic prostatitis are diagnosed when inflammatory cells are present in histological prostate biopsy specimens according to the National Institute of Health (NIH) (14). It was noted that serum PSA was significantly higher in pyuria patients with a urinary leukocyte count exceeding $3 /$ h.p.f. $(\mathrm{p}=0.044)$, but the two groups were similar regarding the cancer detection rate $(p=0.752)$. Histology revealed that pyuria patients had a high inflammation rate, even though clinical symptoms of prostatitis were absent $(\mathrm{p}=0.001)$ (Table 2 ).

Furthermore, NIH-IV prostatitis is often accompanied by prostate hyperplasia $(11,25-27)$. However, the results obtained in this study did not determine that serum CRP was clinically useful in PCa screening. Pyuria patients had higher levels of serum CRP compared with nonpyuria patients (Table 3).

High levels of both PSA and CRP may be due to inflammation. Meanwhile, CRP positive and negative groups did not differ in terms of inflammation rate and cancer detection rate. It was deduced that prostatic inflammation was more reliably signaled by urinary leukocyte count than serum CRP and that serum CRP and elevated PSA were not closely correlated.

According to Tomonori et al., non-palpable PCa and BPH might be differentiated based on an inflammatory index like urinary leukocyte count applied in a clinical setting (25). However, the present study did not support this claim, despite observing that minute prostatic inflammation could be reliably identified based on the urinary leukocyte count. Meanwhile, in a different study conducted on 61 patients displaying PSA in the range 4-10 $\mathrm{ng} / \mathrm{mL}$, normal DRE outcomes and inflammation in expressed prostate secretion, Karazanashvili et al aimed to come up with solutions for making $\mathrm{PCa}$ screening more accurate (28). To that end, they used PSA value modification following treatment with antibiotics as a diagnostic approach. PCa was detected in only $6 \%$ of patients with reduced PSA values, whereas all patients exhibited prostate inflammation. On the other hand, PCa was detected in $83 \%$ of patients with unaltered or ele- 
vated PSA values and prostate inflammation was observed in $17 \%$ of these patients. The authors concluded that PCa screening could be made more accurate by evaluating PSA value modification following treatment with antibiotics since high PSA was determined to a significant extent by chronic prostatitis.

\section{Conclusions}

Male individuals are at high risk of asymptomatic prostatitis if their levels of PSA are high and they present normal DRE outcomes. Prior to biopsy, antibiotics or antiphlogistics should be given to pyuria patients and it is advisable to measure PSA repeatedly as well. In patients without evidence of clinical prostatitis, sterile pyuria should be kept in mind as a cause of increased PSA. Although urine leukocyte count cannot predict non-palpable prostate cancer, it should be incorporated in routine urological evaluation to demonstrate minute prostatic inflammation due to its simplicity, convenience and non-invasiveness.

\section{AUtHOR CONTRIBUTION}

Selamettin Demir: Concept, design, supervision, researches and material, data collection, literature search, writing manuscript, critical review, analysis, interperation.

\section{References}

1. Cooperberg MR, Broering JM, Carroll PR. Risk assessment for prostate cancer metastasis and mortality at the time of diagnosis. $J$ Natl Cancer Inst. 2009; 101:878-887.

2. Pishgar F, Ebrahimi H, Saeedi Moghaddam S, et al. Global, Regional and National Burden of Prostate Cancer, 1990 to 2015: Results from the global burden of disease study 2015. J Urol. 2018; 199:1224-1232.

3. Gann PH. Risk factors for prostate cancer. Rev Urol. 2002; 5:3-10.

4. Hernandez J, Thompson IM. Prostate-specific antigen: a review of the validation of the most commonly used cancer biomarker. Cancer. 2004; 101:894-904

5. Gosselaar C, Roobol MJ, Schröder FH. Prevalence and characteristics of screen-detected prostate carcinomas at low prostate- specific antigen levels: aggressive or insignificant? BJU Int. 2005; 95:231-7.

6. Oesterling JE. Prostate specific antigen: a critical assessment of the most useful tumor marker for adenocarcinoma of the prostate. $J$ Urol. 1991; 145:907-23.

7. Stamey TA, Yang N, Hay AR, et al. Prostate-specific antigen as a serum marker for adenocarcinoma of the prostate. N Engl J Med. 1987; 317:909-16.

8. Marzo AM, Platz EA, Sutcliffe S, et al. Inflammation in prostate carcinogenesis. Nat Rev Cancer. 2007; 7:256-69.

9. Sfanos KS, Hempel HA, De Marzo AM. The Role of inflammation in prostate cancer. Adv Exp Med Biol. 2014; 816:153-81.

10. Palapattu GS, Sutcliffe S, Bastian PJ, et al. Prostate carcinogenesis and inflammation: emerging insights. Carcinogenesis. 2005; 26:1170-81.

11. Morote J, Lopez M, Encabo G, de Torres IM. Effect of inflammation and benign prostatic enlargement on total and percent free serum prostatic specific antigen. Eur Urol. 2000; 37:537-40.
12. Catalona WJ, Southwick PC, Slawin KM, et al. Comparison of percent free PSA, PSA density, and age-specific PSA cutoffs for prostate cancer detection and staging. Urology. 2000; 56:255-60.

13. Brawer MK, Cheli CD, Neaman IE, et al. Complexed prostate specific antigen provides significant enhancement of specificity compared with total prostate specific antigen for detecting prostate cancer. J Urol. 2000; 163:1476-80.

14. Krieger JN, Nyberg L Jr, Nickel JC. NIH consensus definition and classification of prostatitis. JAMA 1999; 282:236-7.

15. Wang MC, Valenzuela LA, Murphy GP, Chu TM. Purification of a human prostate specific antigen. Invest Urol. 1979; 17:159-63.

16. Suzuki H, Akakura K, Igarashi T, et al. Clinical usefulness of serum antip53 antibodies for prostate cancer detection: a comparative study with prostate specific antigen parameters. J Urol. 2004; 171:182-6

17. Renehan AG, Zwahlen M, Minder C, et al. Insulin-like growth factor (IGF) -I, IGF binding protein-3, and cancer risk: systematic review and meta-regression analysis. Lancet. 2004; 363:1346-53.

18. Hersh MR, Knapp EL, Choi J. Newer imaging modalities to assess tumor in the prostate. Cancer Control. 2004; 11:353-7.

19. Inahara M, Suzuki H, Nakamachi $H$, et al. Clinical evaluation of transrectal power doppler imaging in the detection of prostate cancer. Int Urol Nephrol. 2004; 36:175-80.

20. Mantovani A, Allavena P, Sica A, Balkwill F. Cancer related inflammation. Nature. 2008; 454:436-444.

21. De Marzo AM, Platz EA, Sutcliffe S, et al. Inflammation in prostate carcinogenesis. Nat Rev Cancer. 2007; 7:256-269.

22. Kushner I. The phenomenon of the acute phase response. Ann N Y Acad Sci. 1982; 389:39-48.

23. Guthrie GJ, Roxburgh CS, Horgan PG, McMillan DC. Does interleukin-6 link explain the link between tumour necrosis, local and systemic inflammatory responses and outcome in patients with colorectal cancer? Cancer Treat Rev. 2013; 39:89-96.

24. Malinowska K, Neuwirt H, Cavarretta IT, et al. Interleukin-6 stimulation of growth of prostate cancer in vitro and in vivo through activation of the androgen receptor. Endocr Relat Cancer. 2009; 16:155-169.

25. Kato T, Suzuki H, Komiya A, et al. Clinical significance of urinary white blood cell count and serum C-reactive protein level for detection of non-palpable prostate cancer. Int J Urol. 2006; 13:915-919.

26. Stancik I, Lüftenegger W, Klimpfinger M, et al. Effect of NIH-IV prostatitis on free and free-to-total PSA. Eur Urol. 2004; 46:760-4.

27. Irani J, Levillain P, Goujon JM, et al. Inflammation in benign prostatic hyperplasia: correlation with prostate specific antigen value. J Urol. 1997; 157:1301-3.

28. Karazanashvili G, Managadze L. Prostate-specific antigen (PSA) value change after antibacterial therapy of prostate inflammation, as a diagnostic method for prostate cancer screening in cases of PSA value within 4-10 ng/mL and nonsuspicious results of digital rectal examination. Eur Urol. 2001; 39:538-43.

\section{Correspondence}

Selamettin Demir, MD (Corresponding Author)

drselami1978@hotmail.com

The Ministry Of Health, University of Health Sciences, Van Education and Research Hospital, Van (Turkey) 